\title{
Test of understanding of vectors: A reliable multiple-choice vector concept test
}

\author{
Pablo Barniol and Genaro Zavala \\ Physics Education Research and Innovation Group, Department of Physics, Tecnologico de Monterrey, \\ Campus Monterrey, Monterrey 64849, Mexico
}

(Received 20 January 2014; published 17 June 2014)

\begin{abstract}
In this article we discuss the findings of our research on students' understanding of vector concepts in problems without physical context. First, we develop a complete taxonomy of the most frequent errors made by university students when learning vector concepts. This study is based on the results of several test administrations of open-ended problems in which a total of 2067 students participated. Using this taxonomy, we then designed a 20-item multiple-choice test [Test of understanding of vectors (TUV)] and administered it in English to 423 students who were completing the required sequence of introductory physics courses at a large private Mexican university. We evaluated the test's content validity, reliability, and discriminatory power. The results indicate that the TUV is a reliable assessment tool. We also conducted a detailed analysis of the students' understanding of the vector concepts evaluated in the test. The TUV is included in the Supplemental Material as a resource for other researchers studying vector learning, as well as instructors teaching the material.
\end{abstract}

DOI: $10.1103 /$ PhysRevSTPER.10.010121

PACS numbers: 01.40.Fk, 01.40.gb

\section{INTRODUCTION}

Most physical concepts covered in introductory physics courses at the university level are represented by vectors. Therefore, a complete understanding of these physical concepts requires that students have a good grasp of basic vector concepts. In recent years, several researchers have investigated students' understanding of vector concepts. However, we detected three specific issues that still needed to be addressed.

The first was the need to develop a complete taxonomy of the most frequent errors made by university students when learning about vector concepts in introductory physics courses. The second, that to some extent is a consequence of the first, concerns the availability of multiple-choice testing instruments. While several tests that incorporate the recommendations of physics education researchers [1-3] have been created [1,4-12], one that evaluated students' understanding of vector concepts was not yet available. The third issue, suggested by the second, was the need for a large-population (or large sample) study at the university level that would analyze students' understanding of vector concepts after completing their introductory physics courses.

To address these needs, we undertook a research study with three objectives: (1) develop a complete taxonomy of the most frequent errors made when solving vector problems (without a physical context) by university students

Published by the American Physical Society under the terms of the Creative Commons Attribution 3.0 License. Further distribution of this work must maintain attribution to the author(s) and the published article's title, journal citation, and DOI. who have completed the sequence of introductory physics courses, (2) design a reliable multiple-choice test, following the steps recommended by physics education researchers [1-3], that would evaluate students' understanding of vector concepts, and (3) analyze the results and determine how well vector concepts are understood by a large population of university students who have completed the sequence of introductory physics courses. In subsequent sections we will address these objectives, our approach to them, the results of our study, and our analysis. We should note that the test itself has been previously discussed in a short article [13]; additional details are presented here.

\section{PREVIOUS RESEARCH}

Previous studies of students' understanding of vector concepts can be clustered into three groups: (1) those that analyze their understanding of vector concepts in problems without a physical context [14-27], (2) studies that investigate their understanding in problems with a physical context [16-19,24,27-32], and (3) studies that compare students' performance on both types of problems, with and without a physical context [17-19,24,27,31]. Note that some of these studies pertain to more than one group.

The studies in the first group [14-27] are closely related to our investigation, since they analyze students' understanding of vector concepts in problems without a physical context. From this group, six studies [14-19] (by other researchers) identify frequent errors that university students make when they are learning vector concepts. In Table I we show the 10 vector concepts used in introductory courses, and for each concept we note the previous studies that identified the errors that students typically make when 
TABLE I. Ten vector concepts used in the introductory courses. For each concept we note the previous studies that identify students' errors.

\begin{tabular}{|c|c|}
\hline Vector concept & Previous studies \\
\hline 1. Direction of a vector & Knight [14], Nguyen and Meltzer [15] \\
\hline 2. Magnitude of a vector & Knight, Nguyen and Meltzer \\
\hline 3. Component of a vector & Knight, Van Deventer and Wittmann [17], Van Deventer [18] \\
\hline 4. Unit vector in the Cartesian plane & None \\
\hline 5. Graphic representation of a vector & None \\
\hline 6. Vector addition & Knight, Nguyen and Meltzer, Flores et al. [16], Van Deventer [18] \\
\hline 7. Vector subtraction & Flores et al., Van Deventer and Wittmann, Van Deventer [18], Wang and Sayre [19] \\
\hline 8. Scalar multiplication of a vector & Van Deventer [18] \\
\hline 9. Dot product & Knight, Van Deventer and Wittmann, Van Deventer [18] \\
\hline 10. Cross product & Knight, Van Deventer [18] \\
\hline
\end{tabular}

studying that specific concept. The methods used in these studies were individual interviews or tests of open-ended problems. In Sec. V, we discuss the relationship between the previous studies and our research.

As mentioned, in this study we design a multiple-choice test that evaluates students' understanding of vector concepts in problems without a physical context. A previous research study related to ours is by Van Deventer [18]. This author designed isomorphic mathematics and physics multiple-choice vector tests to compare students' performance in both contexts. There are two main differences between the mathematics vector test designed by Van Deventer and our test. The first is that the majority of the distractors (incorrect answers) that Van Deventer builds into his test are based only on interview results from a small population (11 students). In contrast, our distractors were constructed based on the results of multiple testings of open-ended problems, and with a total sample of 2067 students. Secondly, our test evaluates more vector concepts than the Van Deventer study.

\section{METHODS AND TEST OF UNDERSTANDING VECTORS (TUV) DEVELOPMENT}

The research was conducted at a large private Mexican university. The study's participants were students who had finished a calculus-based course on electricity and magnetism $(\mathrm{E} \& \mathrm{M})$. This course is the last of three introductory physics courses taken by students at this institution.

\section{A. Development of the most frequent errors taxonomy}

To develop a complete taxonomy of the most frequent errors that university students make with regard to vector concepts (objective 1), we first conducted several studies over four years that were based on several testings of openended problems. As mentioned, a total of 2067 students of E\&M participated in the study. During this period, the textbooks used in the E\&M course were Physics for Scientists and Engineers by Serway and Jewett [33] and Tutorials in Introductory Physics by McDermott, Shaffer, and the Physics Education Research Group [34]. These studies focused on the detection of frequent student errors and the use of incorrect reasoning and procedures. In designing the open-ended problems, we (1) took into account the results of previous studies [14-19] (Table I), (2) made certain that the problems covered the main vector concepts taught in introductory physics courses at the university level, and (3) ensured that each problem evaluated the core of each vector concept. The results of some of our other studies based on open-ended problems have been reported in previous articles [23-27].

\section{B. Design and evaluation of the TUV}

To design a multiple-choice test that evaluates student understanding of vector concepts (objective 2), we used the results of tests on open-ended problems (as recommended by Beichner [1] and Engelhardt [3]). We designed an initial version of a multiple-choice test and administered it to students at the end of the E\&M course. For most of the items in this first version, we included more than four distractors in order to investigate how frequently each distractor was selected. Following the analysis of this administration, we designed the final version of the 20-item multiple choice test [Test of understanding of vectors (TUV)], which is presented in the Supplemental Material [35]. The content validity of the test's items was evaluated by a panel of experts, in accordance with the procedure recommended by Engelhardt [3]. This test was then administered (in English) to 423 students at the end of the E\&M course. During this period, the textbooks of the E\&M course were Matter and Interaction by Chabay and Sherwood [36] and Tutorials in Introductory Physics [34]. To evaluate the reliability and discriminatory power of the TUV (also objective 2), we followed the procedure suggested by Ding et al. [2].

The TUV tests the 10 vector concepts used in introductory physics courses at the university level, as shown in Tables I and IV. Table IV shows the 10 vector concepts evaluated in the TUV and the items' descriptions. As can be seen in Table IV, only one item each was included for four 
of the 10 vector concepts. It should be noted that we had designed additional associated items, but they were not included on the final version of the TUV because the percentage of correct answers for these questions was higher than that recommended by Ding et al. [2]. For example, for the vector concept "scalar multiplication," we only included the negative scalar multiplication item, since in the open-ended administrations we found that the percentage of correct answers for the positive scalar multiplication problem was higher than the recommended value.

The TUV has 11 items that evaluate the students' understanding of vector concepts posed in graphical form (items 1-5, 9-13, 19), as well as seven items that evaluate their understanding of the calculation of vector concepts $(6,8,14,15,17,18,20)$, and two items that cover graphical and calculations aspects (7 and 16). Figure 1 shows items 13 and 17 of the TUV. Item 13 is an example of the problems in graphical form. Note that for the majority of these items we used a grid, as recommended by Nguyen and Meltzer [15], and we positioned the vectors in a tail-to-tail representation, since some researchers have found that students have more difficulty with this representation [21,24]. Finally, item 17 is an example of the calculation of vector concepts.

\section{Analysis of students' understanding}

To examine the understanding of these vector concepts by a large population of students (objective 3 ), we first analyzed the overall performance of the 423 students in the TUV and clustered the items of the test according to level of difficulty. We then conducted a detailed analysis of the students' understanding of each problem on the TUV. We described the percentages of correct and incorrect answers, and identified the incorrect reasoning and procedures found in the results of tests on open-ended problems. Finally, in the Sec. VI, we classify (for instructional reasons) the most frequent incorrect answer for each of the items of the TUV and point out the incorrect answers that have a frequency proportion of $20 \%$ or greater.

\section{ANALYSIS OF THE TUV}

In designing the TUV, we paid particular attention to issues of content validity, reliability, and discriminatory power. The main results of these analyses are presented here.

\section{A. Content validity}

After the design of the TUV, we checked the content validity of the items of the test. Content validity measures how well the test items cover the content domain they purport to test [3]. In evaluating the TUV, we asked 10 experts (physics faculty members) to rate each item with its corresponding objective ( 1 being low and 5 being high), in accordance with the procedure established by Engelhardt [3]. Each of the items on the TUV was rated as having a high score regarding the match between the test item itself and its stated objectives. The lowest average score for any item was 4.3 and the highest was 5. Moreover, the overall average score was 4.71 . These results are evidence of the high content validity of the TUV.

\section{B. Reliability and discriminatory power}

We also evaluated the reliability and discriminatory power of the TUV, performing the five statistical tests suggested by Ding et al. [2]. The three measures focus on individual test items: the item difficulty index, the item discriminatory index, and the item point biserial. Table II shows these values for each item on the TUV. The other two measures focus on the test as a whole: the

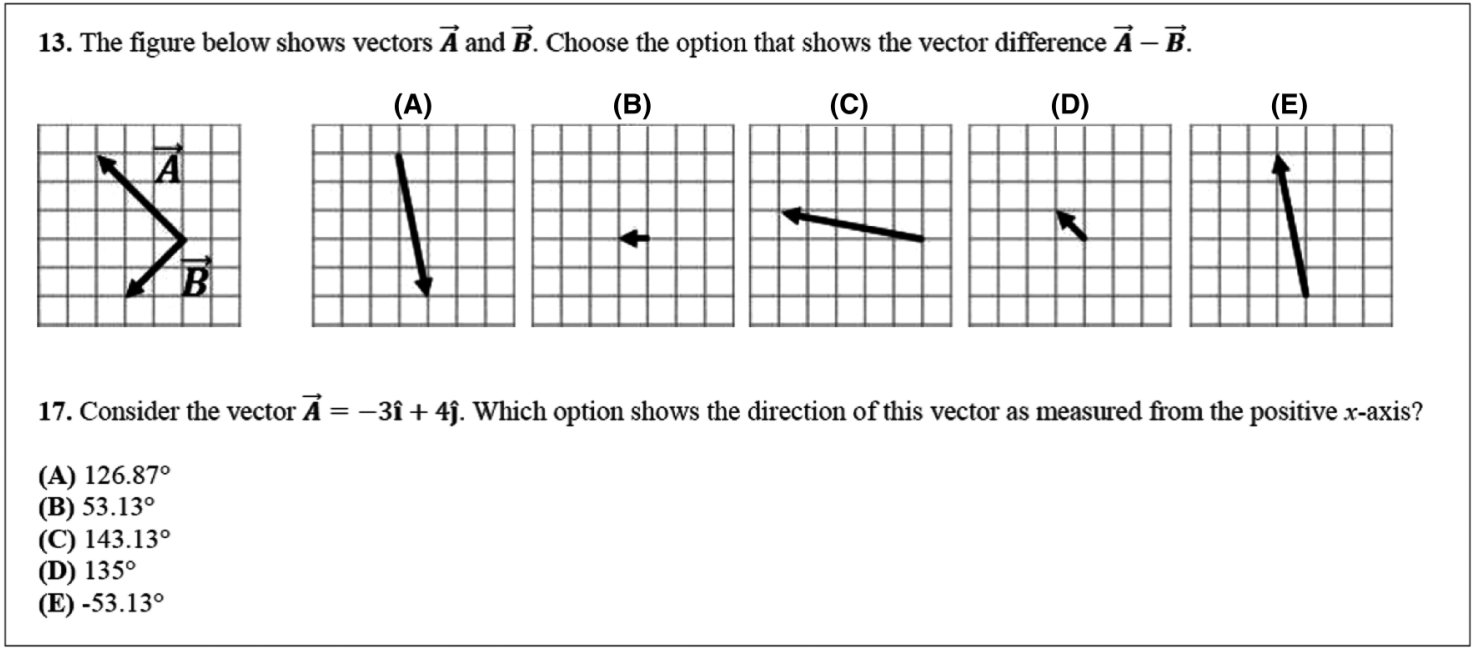

FIG. 1. Item 13 (graphical subtraction of vectors in 2D) and item 17 (calculation of direction of a vector written in unit-vector notation) of the TUV. 
TABLE II. Item difficulty index $(P)$, item discriminatory index $(D)$, and point-biserial coefficient $\left(r_{\mathrm{pbs}}\right)$ for each item of the TUV.

\begin{tabular}{lcccccccccccccccccccc}
\hline \hline & 1 & 2 & 3 & 4 & 5 & 6 & 7 & 8 & 9 & 10 & 11 & 12 & 13 & 14 & 15 & 16 & 17 & 18 & 19 & 20 \\
\hline $\boldsymbol{P}$ & 0.74 & 0.43 & 0.33 & 0.82 & 0.86 & 0.78 & 0.79 & 0.42 & 0.88 & 0.92 & 0.71 & 0.57 & 0.56 & 0.73 & 0.77 & 0.64 & 0.54 & 0.57 & 0.63 & 0.82 \\
$\boldsymbol{D}$ & 0.44 & 0.48 & 0.61 & 0.40 & 0.27 & 0.41 & 0.46 & 0.53 & 0.33 & 0.20 & 0.44 & 0.53 & 0.71 & 0.48 & 0.43 & 0.60 & 0.59 & 0.56 & 0.65 & 0.43 \\
$r_{\mathrm{pbs}}$ & 0.45 & 0.39 & 0.43 & 0.45 & 0.32 & 0.40 & 0.46 & 0.45 & 0.47 & 0.38 & 0.39 & 0.42 & 0.56 & 0.43 & 0.41 & 0.50 & 0.43 & 0.44 & 0.54 & 0.45 \\
\hline \hline
\end{tabular}

Kuder-Richardson reliability test and Ferguson's delta test. We discuss the results of these five statistical tests below.

\section{Item difficulty index}

The item difficulty index $(P)$ is a measure of the difficulty of a single test question. A widely adopted criterion, used by Ding et al. [2], is that the difficulty index should be between 0.3 and 0.9. Table II shows the difficulty index $P$ values for each item on the TUV. The difficulty index values range from 0.33 (item 3 ) to 0.92 (item 10). Only item 10 (graphic representation of a vector in unit-vector notation) has an item difficulty index slightly higher than the desired (0.92). Ding et al. also recommended the calculation of the average difficulty value. The criterion range for the average difficulty value is also [0.3-0.9]. For the TUV, the average difficulty value is 0.68 , which also falls into the suggested range.

\section{Item discriminatory index}

The item discriminatory index $(D)$ is a measure of the discriminatory power of each item on a test. Ding et al. [2] establish two criteria for this index: (1) eliminate items with negative indices and (2) the majority of test items should have a good discrimination index $(D \geq 0.3)$. Table II shows the discrimination index $D$ values for each item on the TUV (using the 25\%-25\% method). We observe that the TUV fulfills these two criteria, since there are no negative items, and the majority of items (18 items) are above 0.3 . The items with an index below this value are items 5 and 10 . From Table II, we note that these items have a high difficulty index, which means that the great majority of students answered these items correctly. This may explain the low discrimination index. We decided to keep these items, since they address important vector concepts taught in introductory physics courses. Ding et al. also recommended the calculation of the average discriminatory index, suggesting a value of $\geq 0.3$. For the TUV the

TABLE III. Summary of the results of the five statistical tests suggested by Ding et al. [2] for the TUV.

\begin{tabular}{|c|c|c|}
\hline Test statistic & Desired values & TUV value \\
\hline Difficulty index & {$[0.3,0.9]$} & Average: 0.68 \\
\hline Discriminatory index & $\geq 0.3$ & Average: 0.48 \\
\hline Point-biserial coefficient & $\geq 0.2$ & Average: 0.44 \\
\hline $\begin{array}{l}\text { Kuder-Richardson } \\
\text { reliability index }\end{array}$ & $\begin{array}{c}\geq 0.7 \text { for group } \\
\text { measures }\end{array}$ & 0.78 \\
\hline Ferguson's delta test & $>0.9$ & 0.97 \\
\hline
\end{tabular}

average discriminatory value is 0.48 (using the 25\%-25\% method), which meets this criterion.

\section{Point-biserial coefficient}

The point-biserial coefficient $\left(r_{\mathrm{pbs}}\right)$ is a measure of the consistency of a single item in relation to the whole test, reflecting the correlation between students' scores on an individual item and their scores on the entire test. A widely adopted criterion, followed by Ding et al. [2], is that an item with a satisfactory point-biserial coefficient must be $r_{\mathrm{pbs}} \geq 0.2$. Table II shows the point-biserial coefficient for each item on the TUV. As one can see, all of the TUV's items satisfy this condition. Ding et al. also recommended the calculation of the average point-biserial coefficient, with a criterion range of $\geq 0.2$. The average coefficient of the TUV is 0.44 , which also fulfills this criterion.

\section{Kuder-Richardson reliability index and Ferguson's delta test}

The Kuder-Richardson reliability index is a measure of the self-consistency of a whole test. Ding et al. [2] state that a test with a reliability index that is higher or equal to 0.7 is reliable for group measures. The index for the TUV is 0.78 , which meets this criterion. Ferguson's delta test measures the discriminatory power of an entire test by investigating how broadly the total scores of a sample are distributed over the possible range. A widely adopted criterion, followed by Ding et al. [2], is that a test with a Ferguson's delta of higher than 0.9 offers a good discrimination. Ferguson's delta test for the TUV is 0.97, which satisfies this requirement.

\section{Summary of the five statistical tests}

We present a summary of the five statistical tests in Table III. From the analysis, we can conclude that the TUV is a reliable test with satisfactory discriminatory power.

\section{STUDENTS' UNDERSTANDING OF VECTOR CONCEPTS, AS INDICATED BY THE TUV RESULTS}

In this section, we analyze the comprehension of vector concepts by students who had finished the introductory physics courses at the university, as demonstrated by their performance on the TUV. Specifically, we studied the results of 423 students who had completed the E\&M course. Table IV shows the 10 vector concepts evaluated 
TABLE IV. The 10 vector concepts evaluated in the TUV, the description of the items, and the percentages of the 423 students of E\&M selecting a particular choice for each item. The correct answer is in boldface and $N$ is for students that did not answer.

\begin{tabular}{|c|c|c|c|c|c|c|c|c|}
\hline Vector concept & Item & Item description & A & B & $\mathrm{C}$ & $\mathrm{D}$ & $\mathrm{E}$ & $\mathrm{N}$ \\
\hline \multirow[t]{2}{*}{ 1. Direction } & 5 & $\begin{array}{l}\text { Choosing a vector with the same direction from among } \\
\text { several in a graph }\end{array}$ & 7 & 2 & 86 & 2 & 3 & 0 \\
\hline & 17 & Calculation of direction of a vector written in unit-vector notation & 54 & 11 & 15 & 11 & 8 & 1 \\
\hline 2. Magnitude & 20 & Calculation of magnitude of a vector written in unit-vector notation & 6 & 82 & 5 & 5 & 1 & 1 \\
\hline \multirow[t]{3}{*}{ 3. Component } & 4 & Graphic representation of $y$ component of a vector & 8 & 3 & 82 & 3 & 4 & 0 \\
\hline & 9 & Graphic representation of $x$ component of a vector & 6 & 3 & 2 & 88 & 1 & 0 \\
\hline & 14 & Calculation of $x$ component of a vector (angle measured from $y$ axis) & 3 & 2 & 73 & 21 & 2 & 0 \\
\hline 4. Unit vector & 2 & Graphic representation of a unit vector & 18 & 33 & 43 & 1 & 5 & 0 \\
\hline 5. Vector representation & 10 & Graphic representation of a vector written in unit-vector notation & 3 & 92 & 1 & 1 & 3 & 更 \\
\hline \multirow[t]{3}{*}{ 6. Addition } & 1 & Graphical addition of vectors in 2D & 8 & 5 & 1 & 12 & 74 & 0 \\
\hline & 7 & $\begin{array}{l}\text { Comparing the vector sum's magnitude of two same-magnitude } \\
\text { vectors at } 90^{\circ} \text { with the magnitude of the vectors. }\end{array}$ & 5 & 79 & 9 & 4 & 3 & 0 \\
\hline & 16 & $\begin{array}{l}\text { Comparing the vector sum's magnitude of two same-magnitude } \\
\text { vectors at } 143.13^{\circ} \text { with the magnitude of the vectors. }\end{array}$ & 12 & 64 & 11 & 4 & 8 & 1 \\
\hline \multirow[t]{2}{*}{ 7. Subtraction } & 19 & Graphical subtraction of vectors in 1D & 0 & 26 & 6 & 4 & 63 & 1 \\
\hline & 13 & Graphical subtraction of vectors in $2 \mathrm{D}$ & 6 & 4 & 8 & 26 & 56 & 0 \\
\hline 8. Scalar multipl & 11 & Graphic representation of a vector multiplied by a negative scalar & 8 & 12 & 71 & 4 & 5 & 0 \\
\hline \multirow[t]{3}{*}{ 9. Dot product } & 3 & Geometric interpretation of dot product as a projection & 27 & 33 & 20 & 9 & 10 & 1 \\
\hline & 6 & Calculation of dot product using the equation $A B \cos \theta$ & 7 & 78 & 3 & 9 & 3 & 0 \\
\hline & 8 & Calculation of dot product of vectors written in unit-vector notation & 42 & 8 & 27 & 5 & 18 & 0 \\
\hline \multirow[t]{3}{*}{ 10. Cross product } & 12 & Geometric interpretation of cross product as a perpendicular vector & 5 & 30 & 5 & 2 & 57 & 1 \\
\hline & 18 & Calculation of a cross product magnitude using the equation $A B \sin \theta$ & 12 & 4 & 6 & 57 & 21 & 0 \\
\hline & 15 & Calculation of cross product of vectors written in unit-vector notation & 77 & 2 & 5 & 15 & 1 & \\
\hline
\end{tabular}

in the TUV, the items' descriptions, and the percentages of the 423 students that selected a particular choice for each item. Note that the percentages of the correct answers correspond to the difficulty indices shown in Table II.

\section{A. Students' overall performance on the TUV}

The average of the scores of the TUV (from the sample of 423 students that finished a calculus-based course on electricity and magnetism) is 13.52 of 20 possible points (each test item is worth 1 point). This average, expressed in percentage of the total possible points, is $68 \%$, which corresponds to the average difficulty index value (0.68) found in the previous section.

The distribution of scores on the TUV is negatively skewed, meaning that the distribution is concentrated on the higher values. For this type of distribution, it is preferable to use the quartile as the measure of spread. The median of the distribution is 14 , the bottom quartile (Q1) is 11 , and the top quartile $(\mathrm{Q} 3)$ is 17 , so the interquartile range is 6 . Note that a distribution that is negatively skewed indicates a less difficult test; however, if we consider that the students are finishing the third introductory physics course (E\&M), and that the TUV evaluates concepts that are frequently used in these courses, it is interesting to note that the students who are on the median (14) had difficulty correctly answering 6 items (out of 20) of the TUV.
Table IV shows the proportion of students correctly answering all items of the TUV. The range of percentages is very wide, from $33 \%$ on item 3 (geometric interpretation of dot product) to $92 \%$ on item 10 (graphic representation of a vector). To analyze these results, we decided to cluster the problems based on the range of proportion of the correct answer.

\section{B. Clusters of TUV items according to level of difficulty}

We classified problems as "high difficulty level" if they had a proportion of correct answers that was equal to or less than $60 \%$, as "medium difficulty level" if they had a correct proportion of $60 \%$ to $80 \%$, and as "low difficulty level" if their proportion of correct answers was equal to or greater than $80 \%$. Table $\mathrm{V}$ shows the division of items by difficulty level.

As shown in Table V, the seven items considered to have a high difficulty level in order of decreasing difficulty are 3

TABLE V. Division of items by difficulty level. Note that the items in each category are arranged in order of difficulty.

\begin{tabular}{lcc}
\hline \hline $\begin{array}{l}\text { Item difficulty } \\
\text { level }\end{array}$ & $\begin{array}{c}\text { Range of correct } \\
\text { answer percentages }\end{array}$ & TUV items \\
\hline High & $\leq 60 \%$ & $3,8,2,17,13,12,18$ \\
Medium & $(60 \%, 80 \%)$ & $19,16,11,14,1,15,6,7$ \\
Low & $\geq 80 \%$ & $4,20,5,9,10$ \\
\hline \hline
\end{tabular}


(geometric interpretation of dot product), 8 (calculation of dot product of vectors written in the unit-vector notation), 2 (graphic representation of unit vector), 17 (calculation of direction of a vector written in unit-vector notation), 13 (graphical subtraction of vector in 2D), 12 (geometric interpretation of cross product), and 18 (calculation of cross product magnitude $A B \sin \theta$ ). It is interesting to note that four of these seven items $(3,8,12$, and 18) evaluate students' understanding of vector products, showing that even after completing the three-course sequence students still have a great deal of difficulty with these operations. The other three items evaluate the unit vector concept, the calculation of direction, and the subtraction in 2D.

The eight items classified as medium difficulty level are $19,16,11,14,1,15,6$, and 7 . The majority of these items (5 out of 8) evaluate vector operations posed in a graphical form: three items evaluate addition (1, 7, and 16), one evaluates subtraction in 1D (item 19), and the last evaluates a negative scalar multiplication of a vector (item 11). It is interesting to note that the other two items in this group are vector product items which are not in the high level group (items 6 and 15), and one item evaluates the calculation of the component of a vector (item 14).

Finally, the five items with a low difficulty level are $4,20,5,9$, and 10 . The great majority of these items (4 out of 5) evaluate vector properties: two evaluate the representation of vector components (items 4 and 9), one evaluates the direction property (item 5), and another evaluates the magnitude of a vector (item 20). Note that the other item of this group evaluates the graphical representation of a vector written in unit-vector notation (item 10), which indirectly evaluates the understanding of the components of a vector.

\section{Students' understanding of each vector concept}

Next, we consider students' understanding of each vector concept evaluated in the TUV, examining their performance on each item separately (Table IV). When analyzing Table IV, we first notice that some distractors on the test have low percentages. This is explained by the fact that the participants in this study were students that were completing the required sequence of university introductory physics courses. We should note that when the test was administered to students just entering the university, the great majority of distractors had percentages that were higher than $5 \%$.

\section{Direction of a vector}

Items 5 and 17 refer to the concept of direction of a vector. In item 5 , students have to choose vectors with the same direction. This item is a modification of an openended problem designed by Nguyen and Meltzer [15]. Eighty-six percent of students answered this item correctly. The most common error (7\%, option A) was to choose vectors $\mathbf{K}$ and $\mathbf{L}$ (error detected by Nguyen and Meltzer).
In our testing of open-ended problems, we found that the students justified this selection by mentioning that vectors $\mathbf{K}$ and $\mathbf{L}$ are pointing to the same "region" (northeast) as vector $\mathbf{A}$, or that vectors $\mathbf{K}$ and $\mathbf{L}$ have positive $x$ and $y$ components like vector A. In a previous article [23], we analyzed the misconceptions about this problem in more detail.

In item 17 , students have to calculate the direction of a vector written in the unit-vector notation $(\mathbf{A}=-3 \mathbf{i}+4 \mathbf{j})$. Knight [14] administered a similar problem but did not catalog the frequent errors. Fifty-four percent of the students answered this item correctly on the TUV. The most common error (15\%, option C) is to state that the direction is $143.13^{\circ}$. In testing the open-ended problems, we found that these students first calculated a $53.13^{\circ}$ angle $\left[\right.$ as $\left.\tan ^{-1}(4 / 3)\right]$ and then incorrectly added a $90^{\circ}$ angle to this value. Another frequent error (11\%, option D) was to select $135^{\circ}$. These students believed that the vector forms an exact negative $45^{\circ}$ angle with the negative $x$ axis. Another error was to choose $53.13^{\circ}$ (11\%, option B), calculating the direction as $\tan ^{-1}(4 / 3)$. And finally, the fourth error was to select $-53.13^{\circ}$ ( $8 \%$, option E), calculating the direction as $\tan ^{-1}(-4 / 3)$.

It is interesting to note the considerable difference in percentage of correct answers that exists between item 5 (86\%) and item 17 (54\%). This shows that students have more difficulty calculating the direction of a vector than selecting vectors with the same direction.

\section{Magnitude of a vector}

Item 20 refers to the magnitude of a vector concept. In this item, students have to calculate the magnitude of a vector written in the unit-vector notation $(\mathbf{A}=2 \mathbf{i}+2 \mathbf{j})$. Knight [14] administered a similar problem but did not itemize the frequent errors. On the TUV, $82 \%$ of students answered this item correctly. The most common error (6\%, option A) was to incorrectly establish that the magnitude of the vector $\mathbf{A}=2 \mathbf{i}+2 \mathbf{j}$ was 2 . When testing the openended problems, we observed that some of the students used an incorrect equation $\sqrt{A_{x}+A_{y}}$ to calculate this magnitude. Another frequent error was to select the unit vector of vector $\mathbf{A}(5 \%)$ as the magnitude of this vector (option D). Another error was to state that the magnitude was 4 (option C, 5\%). Some of the students performed the incorrect calculation of $2^{2}+2^{2}=16$.

\section{Component of a vector}

Items 4,9 , and 14 refer to the component of a vector concept. These items are modifications of multiple-choice problems designed by Van Deventer [18]. The majority of distractors in our items also appear in Van Deventer's problems. The main difference is that we reduced the number of distractors to five, based on the test results of our open-ended problems. In those tests, we also examined the incorrect reasoning used by students that resulted in some 
of the wrong answers. In item 4, students had to choose the correct graphic representation of the $y$ component of a vector. The angle given is from the $y$ axis to the vector. Eighty-two percent of the students answered this item correctly. The most common error (8\%, option A) was to choose a component with a longer magnitude than the correct one. In our open-ended problems, we found that some students justified this selection by stating that the magnitude of the $y$ component of vector $\mathbf{A}$ must have the same value as vector $\mathbf{A}$. Another frequent error (4\%, option E) was to choose a component shorter than the correct one. We found that these students knew the "rule" that components are shorter than the magnitude of the vector but had problems graphically identifying the value of the components.

Item 9 has the same form as item 4, but in this case students had to choose the graphic representation of the $x$ component of a vector. Eighty-eight percent of students answered this problem correctly. The most common error was to choose a component with a longer magnitude than the correct one: $6 \%$ chose option A and 3\% selected option B. The incorrect reasoning found in the open-ended problems was very similar to that established for item 4 . In a previous article [25], we analyzed the misconceptions about items 4 and 9 in greater detail.

In item 14, students had to calculate the $x$ component of a vector. The angle given is from the $y$ axis to the vector. Seventy-three percent of the students answered this item correctly. The most common error (21\%, option D) was to choose $A \cos \varphi$, which is the equation that students memorize in order to calculate the $x$ component. The other incorrect options had lower frequencies. It is interesting to note the difference between the percentages of correct answers for item $14(73 \%)$ and that of items 4 and 9 ( $82 \%$ and $88 \%$, respectively). This shows that students have more difficulty calculating the component of a vector (when the angle given is from the $y$ axis to the vector) than selecting the $x$ and $y$ components of a vector graphically.

\section{Unit vector}

Item 2 is related to the unit vector concept. The question asks students to find the unit vector in the direction of a vector $\mathbf{A}(\mathbf{A}=2 \mathbf{i}+2 \mathbf{j})$. Forty-three percent of the students answered this item correctly (option C). Of those who did not select the correct option, $33 \%$ chose a unit vector that has $x$ and $y$ components of one unit (option B). In our analysis of the related open-ended problem that we had tested earlier, we found that those students choosing option $\mathrm{B}$ believed that this unit vector had a magnitude of 1 . Another incorrect answer that students chose (18\%) was option A: they chose two vectors, the $x$ and $y$ component of vector $\mathbf{A}$. In their reasoning, the students connected the unit vector in the direction of $\mathbf{A}$ with the two components of vector $\mathbf{A}$ written in the unit-vector notation $(2 \mathbf{i}$ and $2 \mathbf{j})$. Five percent of students who chose option E (the same vector $\mathbf{A}$ ) argued that the unit vector of $\mathbf{A}$ was the addition of the two components written in the unit-vector notation, which yields the same vector $\mathbf{A}$. We analyzed this in greater detail in a previous article [26].

\section{Graphic representation of a vector}

Item 10 is a question in which the student is asked for the graphical representation of a vector written in the unitvector notation $(\mathbf{A}=-2 \mathbf{i}+3 \mathbf{j})$. A low proportion of students answered this question incorrectly, since $92 \%$ of them chose option B. Three percent of students chose option A, which is a vector with its tail in $(-2,0)$ and tip in $(0,3)$. In the open-ended problem related to this item, we found that some of these students first sketched the vector components of vector A correctly with their tails in the origin, and then drew the vector from the tip of the $x$ component to the tip of the $y$ component. Three percent of students chose option $\mathrm{E}$ which is a vector with its tail in $(-2,0)$ and tip in $(-2,3)$.

\section{Vector addition}

Items 1,7 , and 16 are related to vector addition. In item 1 , students are asked to calculate the addition of two vectors in two dimensions. The item is a modified version of an open-ended problem designed by Nguyen and Meltzer [15]. Knight [14] and Flores et al. [16] used similar problems in their tests and Van Deventer [18] used it in interviews. In our multiple-choice test, $74 \%$ of students answered the item correctly. Twelve percent of students selected option D, which is a tip-to-tip vector (first reported by Knight [14]). Eight percent of students selected option A, which is a bisector vector, that is, a vector that is in between the two vectors but lacks the precision to be considered a correct sum (error reported by Nguyen and Meltzer [15] and named by Van Deventer [18]). Five percent of students selected a horizontal bisector vector (option B, detected by Nguyen and Meltzer [14]). In a previous article [24], we analyzed in greater detail students' difficulties with open-ended problems similar to item 1.

In item 7, students are asked to compare the magnitude of the vector sum of two perpendicular vectors of the same magnitude to the magnitude of one of the vectors. Nguyen and Meltzer [15] tested a similar problem. Seventy-nine percent of the students answered this question correctly (option B). A large proportion of students answered that the magnitude was the same; however, they used different arguments. Nine percent of students said that it was because both vectors had the same magnitude (option C), 5\% of them chose the option that states that the vector sum only changes direction (option A), and 4\% chose the option that states that the magnitude of the vector sum is the same by means of the direct application of the Pythagorean theorem (option D).

Item 16 is similar to question 7; however, in 16 the angle between the vectors is $143.13^{\circ}$. Flores et al. [16] 
administered a similar open-ended problem. When compared to item 7, a lower proportion of students answered item 16 correctly (64\%). In this question, a large proportion of students answered that the magnitude of the vector sum is greater than the magnitude of one of the vectors. Among this group, $12 \%$ mentioned the use of the Pythagorean theorem (error found by Flores et al.), 11\% justified their answer by saying that the addition of the vectors always yields a resultant vector with a greater magnitude than the individual vectors (option C), and 8\% justified their answer by maintaining that the distance between the tips of the vectors is larger than the magnitude of vector A (option $\mathrm{E}$ ). Only $4 \%$ of students chose option $\mathrm{D}$, that the magnitude of the vector sum is the same as the magnitude of the individual vectors.

\section{Vector subtraction}

Items 13 and 19 refer to vector subtraction. In item 19, students have to choose the vector difference $(\mathbf{A}-\mathbf{B})$ of two vectors $(\mathbf{A}=-3 \mathbf{i} ; \mathbf{B}=5 \mathbf{i})$ in one dimension. Wang and Sayre [19] tested a similar open-ended problem, and Van Deventer [18] included it in interviews. Only 63\% of students answered item 19 correctly. The most frequent error (26\%, option B) was to choose the vector $\mathbf{A}+\mathbf{B}$ as the answer (error detected by Van Deventer). Also, $4 \%$ of students chose the vector $\mathbf{B}-\mathbf{A}$ (option D) and 6\% selected option $\mathbf{C}$, that is, $-(\mathbf{A}+\mathbf{B})$ (these errors had been identified by Wang and Sayre).

In item 13, students have to choose the subtraction vector $(\mathbf{A}-\mathbf{B})$ of two vectors $(\mathbf{A}=-3 \mathbf{i}+3 \mathbf{j}$; $\mathbf{B}=-2 \mathbf{i}-2 \mathbf{j}$ ) in 2D. Flores et al. [16] tested a similar open-ended problem and Van Deventer [18] used it in interviews. Our results indicate that only $56 \%$ of students answered item 13 correctly. The most common error (26\%) was to select vector $-1 \mathbf{i}+1 \mathbf{j}$ (option D). In the open-ended problem that we had previously administered, we found two incorrect procedures: (1) students subtracted the two vectors using components, and incorrectly added the $y$ components of the two vectors, and (2) students incorrectly sketched vector $-\mathbf{B}$ as vector $2 \mathbf{i}-2 \mathbf{j}$ (collinear to vector $\mathbf{A}$ ), adding the vectors graphically to obtain vector $-1 \mathbf{i}+1 \mathbf{j}$. This error has not been reported in the literature for vector subtraction by other researchers. Another frequent error ( $8 \%$, option C) is to choose the vector sum of the two vectors (error detected in interviews by Van Deventer). Another error (6\%, option A) is to choose a vector with opposite direction (error identified by Flores et al.). We found in our testing of open-ended problems that the majority of these students sketched the vector difference directly from the tip of vector $\mathbf{A}$ to the tip of vector $\mathbf{B}$ (tip-to-tip error). Finally, $4 \%$ of students chose a horizontal bisector vector (option B).

\section{Scalar multiplication of a vector}

Item 11 asks students to multiply a negative scalar (negative 3 ) by a vector $(\mathbf{A}=-2 \mathbf{i}+2 \mathbf{j})$. The question is a modified version of a problem used by Van Deventer [18] in interviews. Seventy-two percent of students answered this question correctly. Among the students who did not answer the question correctly, $12 \%$ selected the option representing vector $3 \mathbf{A}$; that is, they had difficulty with the negative sign. Another $8 \%$ of students chose a perpendicular vector with a correct magnitude (option A). In our previous study [26], we found that some students answering this option used an incorrect vector " $\mathbf{A}=-2 \mathbf{i}-2 \mathbf{j}$ " and, therefore, they incorrectly calculated the vector $-3 \mathbf{A}=6 \mathbf{i}+6 \mathbf{j}$. Those students not only had difficulties in identifying the correct vector $\mathbf{A}$, but also in interpreting the scalar multiplication. Five percent of students chose a vector with the correct direction but with an incorrect magnitude (option E), indicating difficulty in interpreting the change in magnitude by the scalar. The remaining $4 \%$ of students chose a vector with a translation of three negative units in the $y$ axis (option D). This error was identified by Van Deventer in his interviews for the positive scalar multiplication. In our previous article [26], we analyzed the misconceptions about item 11 in more detail.

\section{Dot product}

Items 3, 6, and 8 are questions on the dot product. In item 3 , students have to choose the geometric interpretation as a projection of the dot product of two vectors. In interviews on calculating the dot product, Van Deventer [18] found that some students incorrectly consider the dot product of two vectors to be a bisector vector between the vectors. In item 3, only 33\% of students answered the question correctly. A large proportion of students (27\%) chose option A, which indicates that the dot product of two vectors is the magnitude of a vector between the two vectors. In an open-ended problem, we found that the most common incorrect reasoning by these students was to relate the scalar nature of the dot product with the magnitude of a vector. The following is a sample answer by a student who had applied this reasoning: "Dot product is a scalar, that's why its result is a magnitude and not a vector." Another large proportion of students (20\%) chose option C, the bisector vector between the vectors. In the open-ended problem related to this item, we detected, as did Van Deventer [18], that the most common incorrect reasoning by these students was to associate the dot product with addition vectors. Also, $10 \%$ of students thought that the dot product was a horizontal vector in the direction of vector $\mathbf{D}$. We found that the most common incorrect reasoning for this error was based on an incorrect calculation of the dot product using unit-vector notation. A representative student answer that illustrates this incorrect reasoning is "To get the dot product, one multiplies $A \cdot B=(x \boldsymbol{i}+x \boldsymbol{j}) \cdot(y \boldsymbol{i})=x y \boldsymbol{i}$. As can be seen, $\boldsymbol{i} \cdot \boldsymbol{j}$ cancel each other out and only the multiplication $\boldsymbol{i} \cdot \boldsymbol{i}$ remains." Finally, 9\% of students stated 
that the dot product was a perpendicular vector (confusing the dot product with the cross product).

In item 6, students have to calculate the dot product of two vectors that form an angle $\theta$ between them. Knight [14] administered a similar problem but did not catalog the frequent errors. Seventy-eight percent of students answered this question correctly. Nine percent of students chose option D, i.e., $A B \sin \theta$, which is the expression for calculating the magnitude of the cross product of two vectors. Seven percent of students selected $A B$ (option A), which is the direct multiplication of the magnitudes of the vectors. In previous publications [25,27], we analyzed the misconceptions about items 3 and 6 in more detail.

In item 8, students have to calculate the dot product of two vectors written in the unit-vector notation $(\mathbf{A}=$ $1 \mathbf{i}+3 \mathbf{j}$ and $\mathbf{B}=5 \mathbf{i}$ ). Knight [14] tested a similar problem (without identifying the most frequent errors made by students), and Van Deventer [18] included it in interviews. In our results, only $42 \%$ of students answered item 8 correctly (option A). A large proportion of students (27\%) chose $5 \mathbf{i}+3 \mathbf{j}$ (option $\mathrm{C}$ ). In the open-ended problem that we had previously administered, we found that these students calculated a dot product of vector $1 \mathbf{i}$ (from vector A) and vector $5 \mathbf{i}$ (from vector $\mathbf{B}$ ), incorrectly obtaining the vector $5 \mathbf{i}$, and then added the vector $3 \mathbf{j}$ (from vector $\mathbf{B}$ ) to the result. Another large proportion of students (18\%) answered 5i (option E). These students performed the same calculation as the previous ones, without adding the $3 \mathbf{j}$ (from vector B) to the vector 5i. Eight percent of students chose option B $(-15 \mathbf{k})$, which is the cross product of the two vectors, and the rest $(8 \%)$ selected option $\mathrm{D}(6 \mathbf{i}+3 \mathbf{j})$, which is the addition of the two vectors.

It is interesting to note the great difference between the percentage of correct answers for item $6(78 \%)$, item 3 $(33 \%)$, and item $8(42 \%)$. This shows that students have more difficulty interpreting and calculating the dot product in the unit-vector notation than correctly applying the equation $A B \cos \theta$.

\section{Cross product}

Items 12,15 , and 18 are questions regarding the cross product. In item 12, students have to choose the best geometric interpretation of the cross product of two vectors from among several options. In a previous study [18], Van Deventer used a similar problem in student interviews, but in that study students were asked to describe the direction of the cross product. This question assumed that students knew that the result of the product was a vector, and we tried to confirm this supposition. On our test, $57 \%$ of students answered item 12 correctly. The most common mistake (30\%, option B) was to choose a vector with an opposite direction. The rest of the students chose other responses, such as option A (5\%), which states that the cross product is a vector between the two vectors in the product (identified by Van Deventer in student interviews).
Others chose option $\mathrm{C}(5 \%)$, indicating that they believe that the cross product is the magnitude of a vector between the two vectors in the product.

In item 18, students have to calculate the magnitude of the cross product of two vectors that form an angle $\theta$. Knight [14] tested a similar problem (without identifying the most frequent errors made by students), and Van Deventer [18] included it in interviews. Fifty-seven percent of students answered item 18 correctly. The most frequent error $(21 \%)$ was option $\mathrm{E}, A B \cos \theta$, the equation used to calculate the dot product (this error was also identified by Van Deventer in his interviews). The second most common error $(12 \%)$ was option $\mathrm{A}, A \cos \theta B \sin \theta$. We found that these students correctly calculated the $x$ component of vector $\mathbf{A}$ as $A \cos \theta$ but incorrectly calculated the $x \operatorname{compo-}$ nent of vector $\mathbf{B}$ as $B \sin \theta$. They then multiplied these two expressions. Six percent of students selected option $\mathrm{C}$, $A B \sin \left(90^{\circ}-\theta\right)$, and $4 \%$ chose the equation $A B$ (option $\mathrm{B}$ ), which is the direct multiplication of the magnitudes of the two vectors. In a previous publication [25], we analyzed the misconceptions about items 12 and 18 in more detail.

In item 15, students have to calculate the cross product of two vectors using the unit-vector notation $(\mathbf{A}=1 \mathbf{i}+3 \mathbf{j}$ and $\mathbf{B}=5 \mathbf{i})$. Seventy-seven percent answered item 15 correctly. The most common error was option D (15\%), which has the opposite sign $(15 \mathbf{k})$. The second most common error (5\%) was option C, $5 \mathbf{i}+3 \mathbf{j}$. We found that these students calculated a product of vector $1 \mathbf{i}$ (from vector $\mathbf{A})$ and $5 \mathbf{i}$ (from vector $\mathbf{B}$ ), incorrectly obtaining vector $5 \mathbf{i}$. They then added vector $3 \mathbf{j}$ (from vector $\mathbf{B}$ ).

It is interesting to note the considerable difference that exists between the proportion of correct answers for item 15 (77\%) and that of items 12 and 18 (57\% for both). This shows that students have more difficulties in interpreting the cross product and calculating its magnitude as $A B \sin \theta$ than in correctly calculating the cross product in unit-vector notation.

If we observe the items in which students have to do calculations with two vectors written in unit-vector notation, we note that students have more difficulties with the dot product (item 8, 42\% correct) than with the cross product (item 15, 77\% correct). This difference seems to be due to the fact that students have more difficulties with the dot product interpretation (item 3, 33\% correct) than with the cross product interpretation (item 12, 57\% correct). As discussed, a significant proportion of students mistakenly think that the dot product is a vector. Therefore, this error may also have an effect in item 8 in which all the incorrect options are vectors.

In contrast, in the items in which the definitions with trigonometric functions are used, students have more difficulties with the cross product (item 18, 57\% correct) than with the dot product (item $6,78 \%$ correct). This difference could be due to a generalization of the students that the product between two vectors is $A B \cos \theta$. In the dot product item, $78 \%$ of students answer that which is correct and only 
9\% of students select the incorrect option $A B \sin \theta$. On the other hand, in the cross product item, a large proportion of students (21\%) select $A B \cos \theta$, which in this case is incorrect.

\section{DISCUSSION}

In this section we focus, for instructional reasons, on the most frequent incorrect answer for each of the TUV's items. First, we classify them by type. We then display a table of those incorrect answers that have a frequency proportion that is equal to or greater than $20 \%$. Next we perform analyses of both.

\section{A. Classification of the most frequent incorrect answer for each of the items of the TUV}

The most frequent incorrect answer for each of the items of the TUV can be clustered into four groups. Table VI presents this classification.
As we can see from Table VI, the first group of difficulties is in the graphical properties of direction, magnitude, and components of a vector. The frequent answers to five items on the test (items 5, 4, 9, 2, and 7) are represented in this group. Upon analyzing these answers, two categories of errors emerge. The first category involves understanding the concept of direction (item 5). As established in Sec. V, students have difficulties relating the direction of a vector with the angle it forms with the $x$ axis, and instead relate it to the region where it points. The second category of error is found in the remaining items $(4,9,2$, and 7$)$. As we can see, students mistakenly believe that the $x$ and $y$ components of a vector have the same value as the magnitude of the vector. It is important to note that item 7 concerns vector addition of vectors at $90^{\circ}$ and does not refer explicitly to vector components; the situation is completely analog.

TABLE VI. Classification of the most frequent incorrect answer for each of the items of the TUV. Note that the most frequent incorrect answer of the dot product interpretation problem (item 3) cannot be classified in a specific group because of the peculiar nature of the dot product operation.

\begin{tabular}{|c|c|c|}
\hline Difficulties group & Description & Most frequent incorrect answer for items in this group \\
\hline Graphical properties & $\begin{array}{l}\text { Difficulties in understanding the } \\
\text { graphical properties of direction, } \\
\text { magnitude, and components of a } \\
\text { vector. }\end{array}$ & $\begin{array}{l}\text { Item 5: Two vectors that form different angles with the } x \text { axis but pointing } \\
\text { to the same region (northeast) have the same direction. } \\
\text { Item 4: The } y \text { component of a vector has the same value as the magnitude } \\
\text { of the vector. } \\
\text { Item 9: The } x \text { component of a vector has a longer magnitude than the correct } \\
\text { one, because it has the same "value" as the magnitude of the vector. } \\
\text { Item 2: A unit vector has } x \text { and } y \text { components of one unit. } \\
\text { Item 7: The magnitude of the vector sum of two same-magnitude vectors } \\
\text { at } 90^{\circ} \text { is the same as the magnitude of the vectors. }\end{array}$ \\
\hline Graphical procedures & $\begin{array}{l}\text { Difficulties in understanding the } \\
\text { graphical procedures of vector } \\
\text { operations: addition, negative } \\
\text { scalar multiplication, and cross } \\
\text { product. }\end{array}$ & $\begin{array}{l}\text { Item 1: Tip-to-tip error in the addition of two vectors. } \\
\text { Item 10: Sketching vector }-2 \mathbf{i}+3 \mathbf{j} \text { from the tip of the } x \text { component to the } \\
\text { tip of the } y \text { component. } \\
\text { Item 11: Vector with incorrect opposite direction in the negative scalar } \\
\text { multiplication. } \\
\text { Item 19: Add vectors in the subtraction of two vectors in } 1 \mathrm{D} \text {. } \\
\text { Item 13: Represent the subtraction vector }(\mathbf{A}-\mathbf{B}) \text { of } \mathbf{A}=-3 \mathbf{i}+3 \mathbf{j} \text { and } \\
\mathbf{B}=-2 \mathbf{i}-2 \mathbf{j} \text { as }-1 \mathbf{i}+1 \mathbf{j} \text { (not }-1 \mathbf{i}+5 \mathbf{j}) \text {. } \\
\text { Item } 12 \text { : Vector with incorrect opposite direction in the cross product of } \\
\text { two vectors. }\end{array}$ \\
\hline $\begin{array}{l}\text { Geometric calculation } \\
\text { procedures }\end{array}$ & $\begin{array}{l}\text { Difficulties with calculations that } \\
\text { involve angles, trigonometric } \\
\text { functions, and the Pythagorean } \\
\text { theorem }\end{array}$ & $\begin{array}{l}\text { Item 17: Calculate the direction of vector } \mathbf{A}=-3 \mathbf{i}+4 \mathbf{j} \text { as } 143.13^{\circ} \text { (not } \\
\text { 126.87 } \text { ). } \\
\text { Item 14: Use the cosine function to calculate the } x \text { component of a vector } \\
\text { when the angle given is measured from the } y \text { axis. } \\
\text { Item 6: Calculate the dot product as } A B \sin \theta \text {. } \\
\text { Item 18: Calculate the magnitude of a cross product as } A B \cos \theta \text {. } \\
\text { Item 16: Apply the Pythagorean theorem incorrectly to calculate the } \\
\text { magnitude of the vector sum of two vectors at } 143.13^{\circ} \text {. } \\
\text { Item 20: Apply the Pythagorean theorem incorrectly when calculating the } \\
\text { magnitude of vector } 2 \mathbf{i}+2 \mathbf{j} \text { as } 2 .\end{array}$ \\
\hline $\begin{array}{l}\text { Unit-vector notation } \\
\text { calculation } \\
\text { procedures }\end{array}$ & $\begin{array}{l}\text { Difficulties with calculations of dot } \\
\text { and cross products that involve } \\
\text { unit-vector notation. }\end{array}$ & $\begin{array}{l}\text { Item 8: Calculate the dot product }(\mathbf{A} \cdot \mathbf{B}) \text { of the vectors } \mathbf{A}=1 \mathbf{i}+3 \mathbf{j} \text { and } \\
\quad \mathbf{B}=5 \mathbf{i} \text { as } 5 \mathbf{i}+3 \mathbf{j} \text {. } \\
\text { Item } 15: \text { Calculate the cross product }(\mathbf{A} \times \mathbf{B}) \text { of the vectors } \mathbf{A}=1 \mathbf{i}+3 \mathbf{j} \\
\text { and } \mathbf{B}=5 \mathbf{i} \text { as a vector with opposite sign }(15 \mathbf{k}) \text {. }\end{array}$ \\
\hline
\end{tabular}


The second group of difficulties in Table VI is in the graphical procedures of vector operations. The frequent answers for six items on the test (items 1, 10, 11, 19, 13, and 12) fall into this group. Here we find three categories of errors. The first category is in a vector addition operation, in which students make the tip-to-tip error (items 1 and 10). This error is found in problem 2D (item 1), where we see that students sketch the vector sum $\mathbf{A}+\mathbf{B}$ from the tip of vector $\mathbf{B}$ to the tip of vector $\mathbf{A}$. It also appears in item 10, the problem that asks students to sketch vector $\mathbf{A}=-2 \mathbf{i}+3 \mathbf{j}$; in their responses, some students sketched vector $\mathbf{A}$ from the tip of vector $-2 \mathbf{i}$ (with its tail in the origin) to the tip of vector $3 \mathbf{j}$ (also with its tail in the origin). The second category of errors that emerges is in the representation of the negative multiplication of a vector. Students had difficulty with item 11 (negative scalar multiplication of a vector) and items 19 and 13 (subtraction of vector in $1 \mathrm{D}$ and 2D). In items 11 and 19, they failed to invert the direction of the vectors multiplied by -1 , since in item 11 they chose a vector pointing in the opposite direction, and in item 19 they added the vectors instead of subtracting them. In item 13 , students also had difficulties with the negative of vector $\mathbf{B}(\mathbf{B}=-2 \mathbf{i}-2 \mathbf{j})$, since they represented it in their subtraction procedures as a "perpendicular" vector $(2 \mathbf{i}-2 \mathbf{j})$. Finally, the third category of errors stems from an incorrect application of the righthand rule. As we can see from the cross product problem in Table VI (item 12), students chose the option with the incorrect opposite direction.

The third group of student difficulties classified in Table VI illustrates problems with geometric calculations. The frequent answers to five test items (items 17, 14, 6, 18, 16 , and 20) fall into this group. Three categories of errors emerge from the analysis of these answers. The first category is made up of calculations that involve angles. This error is found in item 17, in which students incorrectly calculated the direction of vector $\mathbf{A}=-3 \mathbf{i}+4 \mathbf{j}$ as $143.13^{\circ}$ (not $126.87^{\circ}$ ). As mentioned in Sec. V, these students had difficulties with supplementary angle calculations, since

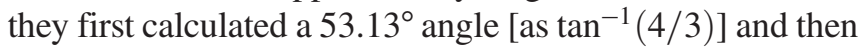
incorrectly added a $90^{\circ}$ angle to this value. The second category of errors is found in items 14, 6, and 18, where calculations of trigonometric functions (sine and cosine) are involved. In item 14, students incorrectly calculated the $x$ component of a vector when the angle given is measured from the $y$ axis using the cosine function instead of the sine function. In item 6 , in order to calculate the dot product, students incorrectly applied the sine function instead of the cosine function, and in item 18, when calculating the magnitude of the cross product of two vectors, students incorrectly applied the cosine function instead of the sine function. As we see, students had difficulty selecting which of the two functions needed to be used in these situations. Finally, in the third category of this classification group, students had difficulty using the Pythagorean theorem in two of the items. In item 16, students used the Pythagorean theorem incorrectly to calculate the magnitude of the vector sum of two vectors at $143.13^{\circ}$, showing that they do not know the requirements for using this theorem, and in item 20 , they incorrectly applied it by calculating the magnitude of vector $2 \mathbf{i}+2 \mathbf{j}$ as 2 .

The fourth group of student difficulties is in calculations that involve unit-vector notation. The most frequent incorrect answers for vector products are shown in this group. As mentioned in Sec. V, in item 8 students incorrectly calculated the dot product of the vectors $\mathbf{A}=1 \mathbf{i}+3 \mathbf{j}$ and $\mathbf{B}=5 \mathbf{i}$ as $5 \mathbf{i}+3 \mathbf{j}$, and in item 15 they incorrectly calculated the cross product of the vectors $\mathbf{A}=1 \mathbf{i}+3 \mathbf{j}$ and $\mathbf{B}=5 \mathbf{i}$ as a vector with the opposite sign $(15 \mathbf{k})$. These errors seem to originate in the procedures that the students followed. It is interesting to note that in the TUV there are three other items $(10,17$, and 20) that use the unit-vector notation, but as shown above these errors seem to have their origin in other specific difficulties.

Finally, we must note that the most frequent incorrect answer for the dot product interpretation problem (item 3) cannot be clustered in a specific classification group. This is due to the peculiar nature of the dot product operation. Remember that this operation is performed between two vectors and the result is a scalar, not a vector. As noted in Sec. V, in item 3 the most common incorrect answer was to interpret the dot product of two vectors as the magnitude of a vector between the vectors.

\section{B. Identification of incorrect answers with higher frequency percentages}

In the latter analysis we classified the most frequent incorrect answer for each of the items on the TUV. However, the percentages of these answers vary widely. For example, in the unit vector representation problem (item 2), the most common incorrect answer has a frequency rate of $33 \%$, while that of the graphic representation of the $y$ component of a vector (item 4) is only $8 \%$. Both for this reason and for instructional purposes, we decided to identify the incorrect answers on the TUV that have a frequency rate of equal to or greater than $20 \%$. Table VII shows the nine incorrect answers that fulfill this criterion.

Table VII reveals several facts that are worth noting. Five of the nine errors (errors 2, 3, 4, 7, and 9) are in vector products and two of these five errors (errors 3 and 9) are in the interpretation of the dot product problem. Also, two of the nine errors (errors 5 and 6 ) are in the representation of vector subtraction. Finally, the other two errors are in the representation of a unit vector (error 1) and the calculation of the $x$ component of a vector (error 8).

An analysis of the errors in Table VII to determine where they fall in the four difficulties groups shown in Table VI yielded interesting results. We observe that the errors are not concentrated in a specific group, but rather are found in different groups: error 1 is in the graphical properties 
TABLE VII. Incorrect answers on the TUV with frequency rates of equal to or greater than $20 \%$. (Answers are in order of decreasing percentage.)

\begin{tabular}{|c|c|c|c|c|}
\hline Error & Item & Option & Error description & $\%$ \\
\hline 1 & 2 & $\mathrm{~B}$ & Consider that a unit vector has $x$ and $y$ components of one unit. & $33 \%$ \\
\hline 2 & 12 & $\mathrm{~B}$ & Vector with incorrect opposite direction in the cross product of two vectors. & $30 \%$ \\
\hline 3 & 3 & A & $\begin{array}{l}\text { Interpret the dot product of two vectors as the magnitude of a vector between } \\
\text { the two vectors. }\end{array}$ & $27 \%$ \\
\hline 4 & 8 & $\mathrm{C}$ & Calculate the dot product $(\mathbf{A} \cdot \mathbf{B})$ of the vectors $\mathbf{A}=1 \mathbf{i}+3 \mathbf{j}$ and $\mathbf{B}=5 \mathbf{i}$ as $5 \mathbf{i}+3 \mathbf{j}$. & $27 \%$ \\
\hline 5 & 13 & $\mathrm{D}$ & $\begin{array}{l}\text { Represent the subtraction vector }(\mathbf{A}-\mathbf{B}) \text { of } \mathbf{A}=-3 \mathbf{i}+3 \mathbf{j} \text { and } \\
\mathbf{B}=-2 \mathbf{i}-2 \mathbf{j} \text { as }-1 \mathbf{i}+1 \mathbf{j}(\text { not }-1 \mathbf{i}+5 \mathbf{j}) \text {. }\end{array}$ & $26 \%$ \\
\hline 6 & 19 & $\mathrm{~B}$ & Add vectors in the subtraction of two vectors in $1 \mathrm{D}$ & $26 \%$ \\
\hline 7 & 18 & $\mathrm{E}$ & Calculate the magnitude of a cross product as $A B \cos \theta$. & $21 \%$ \\
\hline 8 & 14 & $\mathrm{D}$ & $\begin{array}{l}\text { Use the cosine function to calculate the } x \text { component of a vector when the } \\
\text { angle given is measured from the } y \text { axis. }\end{array}$ & $21 \%$ \\
\hline 9 & 3 & $\mathrm{C}$ & Interpret the dot product of two vectors as a vector between the two vectors. & $20 \%$ \\
\hline
\end{tabular}

group; errors 2, 5, and 6 are in the graphical procedures group; errors 7 and 8 are in the geometric calculation procedures group; error 4 is in the unit-vector notation group; and, finally, errors 3 and 9 are related to difficulties in interpreting the dot product that are not classified in a specific group.

\section{CONCLUSIONS}

The first objective of this study was to develop a complete taxonomy of the most frequent errors that university students continue to make with regard to the vector concepts after completing their introductory physics courses. We developed this taxonomy by taking into consideration the results of several studies that were based on tests of opened-ended problems; we then designed the TUV test with this taxonomy in mind. The test reflects this taxonomy, since the distractor (incorrect answer) for each item on the TUV corresponds to a frequent error that students make when applying that specific vector concept. In the analysis of students' responses to the TUV test questions, we detailed the characteristics of each error and specified the incorrect reasoning or procedure (found in the results of tests on open-ended problems) that led them to commit this error. This taxonomy has three characteristics that need to be emphasized: it includes the basic vector concepts used in introductory physics courses; it references errors and incorrect reasoning that have not been previously reported in the literature; and it summarizes, to a certain extent, the previous studies that analyze the students' difficulties with vector concepts. Finally, it is important to note that this taxonomy could be used by physics education researchers to design new instructional materials that will explicitly address the more persistent conceptual difficulties [37].

The second objective of the study was to design a reliable multiple-choice test, following the steps recommended by physics education researchers [1-3], that would evaluate students' understanding of vectors. As mentioned, we designed the TUV test using the aforementioned taxonomy, and its content validity was assessed by a panel of experts. We then evaluated the reliability and discriminatory power of the TUV test, following the procedure suggested by Ding et al. [2], and concluded that the TUV test is a reliable tool with satisfactory discriminatory power. It is noteworthy that the TUV is the first test for evaluating students' understanding of vector concepts that fulfills these criteria. The test could be used to analyze students' understanding of vector concepts in different institutions, to investigate students' learning gains [38,39] although pretest results could be very low as with other standardized tests, like in electricity and magnetism [7] in which students are not familiar with the concepts, and to test the effectiveness of new instructional material based on research designed to increase student knowledge and understanding [38,39]. Some physics teachers may wonder if certain items could be eliminated from the test in order to suit their curriculum. For example, teachers of algebra-based physics courses may wish to eliminate questions related to the dot and cross product. Since the great majority of items on the TUV fulfill the criteria for item reliability and discriminatory power established by Ding et al. [2], it is very probable that a test without some of these items will still fulfill all the criteria.

The third objective of this study was to analyze the understanding of vector concepts by a large population of students upon completing the sequence of introductory physics courses at the university level. We performed this analysis by administering the TUV to 423 such students. First, we analyzed the students' overall performance on the test, finding that those at the median level had difficulty in correctly answering 6 of the items on the test. We also found that they had further difficulties with 7 of the 20 items (items 3, 8, 2, 17, 13, 12, 18). These items evaluate the geometric interpretation of dot product, the calculation of dot product of vectors written in the unit-vector notation, the graphic representation of unit vector, the calculation of direction of a vector written in unit-vector notation, the graphical subtraction of vector in $2 \mathrm{D}$, the geometric 
interpretation of cross product, and the calculation of cross product magnitude as $A B \sin \theta$.

We also analyzed in detail the students' understanding of each problem on the TUV, describing the percentages of correct and incorrect answers, and identifying the incorrect reasoning and procedures. Finally, in Sec. VI we developed, for instructional purposes, a classification of the most frequent incorrect answer for each of the items on the TUV, categorizing them in four groups by type of difficulty: (1) graphical properties, (2) graphical procedures, (3) geometric calculation procedures, and (4) unitvector notation calculation procedures. We also identified and analyzed the nine incorrect answers that had a frequency proportion of equal to or greater than $20 \%$. These analyses of students' understanding share the special characteristic that they are the first in the literature to have been carried out using a multiple-choice test that fulfills the previously specified criteria. We believe that these analyses provide physics teachers and researchers with a general description of the difficulties that university students have when applying vector concepts, even after completing their introductory physics courses, and could guide the design of new instructional material intended to increase students' understanding of vector concepts.

\section{ACKNOWLEDGMENTS}

The authors acknowledge the support received from Tecnologico de Monterrey through Grant No. CAT140.
[1] R. Beichner, Testing student interpretation of kinematic graphs, Am. J. Phys. 62, 750 (1994).

[2] L. Ding, R. Chabay, B. Sherwood, and R. Beichner, Evaluating an electricity and magnetism assessment tool: Brief electricity and magnetism assessment, Phys. Rev. ST Phys. Educ. Res. 2, 010105 (2006).

[3] P. Engelhardt, An Introduction to Classical Test Theory as Applied to Conceptual Multiple-Choice Tests in Getting Started in PER, Vol. 2, http://www.compadre.org/ Repository/document/ServeFile.cfm? $\mathrm{ID}=8807 \& \mathrm{DocID}=1148$.

[4] D. Hestenes, M. Wells, and G. Swackhamer, Force Concept Inventory, Phys. Teach. 30, 141 (1992).

[5] R. Thornton and D. Sokoloff, Assessing student learning of Newton's laws: The Force and Motion Conceptual Evaluation and the evaluation of active learning laboratory and lecture curricula, Am. J. Phys. 66, 338 (1998).

[6] C. Singh and D. Rosengrant, Multiple-choice test of energy and momentum concepts, Am. J. Phys. 71, 607 (2003).

[7] D. Maloney, T. L. O'Kuma, C. J. Hieggelke, and A. Van Heuvelen, Surveying students' conceptual knowledge of electricity and magnetism, Am. J. Phys. 69, S12 (2001).

[8] P. Engelhardt and R. Beichner, Students' understanding of direct current resistive electrical circuits, Am. J. Phys. 72, 98 (2004).

[9] A. Tongchai, M. D. Sharma, I. D. Johnston, K. Arayathanitkul, and C. Soankwan, Developing, evaluating and demonstrating the use of a conceptual survey in mechanical waves, Int. J. Sci. Educ. 31, 2437 (2009).

[10] S. Wuttiprom, M. D. Sharma, I. D. Johnston, R. Chitaree, and C. Soankwan, Development and use of a conceptual survey in introductory quantum physics, Int. J. Sci. Educ. 31, 631 (2009).

[11] S. B. McKagan, K. K. Perkins,, and C. E. Wieman, Design and validation of the quantum mechanics conceptual survey, Phys. Rev. ST Phys. Educ. Res. 6, 020121 (2010).
[12] J. S. Aslanides and C. M. Savage, Relativity concept inventory: Development, analysis, and results, Phys. Rev. ST Phys. Educ. Res. 9, 010118 (2013).

[13] P. Barniol and G. Zavala, in Proceedings of the Physics Education Research Conference 2013, Portland, OR, 2014, edited by P. V. Engelhardt, A. D. Churukian, and D. L. Jones, http://www.compadre.org/Repository/ document/ServeFile.cfm?ID=13098\&DocID=3633.

[14] R. D. Knight, Vector knowledge of beginning physics students, Phys. Teach. 33, 74 (1995).

[15] N. Nguyen and D. Meltzer, Initial understanding of vector concepts among students in introductory physics courses, Am. J. Phys. 71, 630 (2003).

[16] S. Flores, S. E. Kanim, and C. H. Kautz, Student use of vectors in introductory mechanics, Am. J. Phys. 72, 460 (2004).

[17] J. Van Deventer and M. C. Wittmann, Comparing student use of mathematical and physical vector representations, AIP Conf. Proc. 951, 208 (2007).

[18] J. Van Deventer, Master's thesis, The University of Maine, 2008.

[19] T. Wang and E. C. Sayre, Maximum likelihood estimation (MLE) of students' understanding of vector subtraction, AIP Conf. Proc. 1289, 329 (2010).

[20] J. M. Hawkins, J. R. Thompson,, and M. C. Wittmann, Students' consistency of graphical vector addition method on 2-D vector addition tasks, AIP Conf. Proc. 1179, 161 (2009).

[21] J. M. Hawkins, J. R. Thompson, M. C. Wittmann, E. C. Sayre, and B. W. Frank, Students' responses to different representations of a vector addition question, AIP Conf. Proc. 1289, 165 (2010).

[22] B. E. Hinrichs, Writing position vectors in 3-d space: A student difficulty with spherical unit vectors in intermediate E\&M, AIP Conf. Proc. 1289, 173 (2010).

[23] P. Barniol and G. Zavala, Investigation of students' preconceptions and difficulties with the vector direction 
concept at a Mexican university, AIP Conf. Proc. 1179, 85 (2009).

[24] P. Barniol and G. Zavala, Vector addition: Effect of the context and position of the vectors, AIP Conf. Proc. 1289, 73 (2010).

[25] G. Zavala and P. Barniol, Students' understanding of the concepts of vector components and vector products, AIP Conf. Proc. 1289, 341 (2010).

[26] P. Barniol and G. Zavala, Students' difficulties with unit vectors and scalar multiplication of a vector, AIP Conf. Proc. 1413, 115 (2012).

[27] G. Zavala and P. Barniol, Students' understanding of dot product as a projection in no-context, work and electric flux problems, AIP Conf. Proc. 1513, 438 (2013).

[28] J. M. Aguirre, Students' conceptions about the vector characteristics of three physics concepts, J. Res. Sci. Teach. 21, 439 (1984).

[29] J. M. Aguirre, Student preconceptions about vector kinematics, Phys. Teach. 26, 212 (1988).

[30] J. M. Aguirre and G. Rankin, College students' conceptions about vector kinematics, Phys. Educ. 24, 290 (1989).

[31] P.S. Shaffer and L.C. McDermott, A research-based approach to improving student understanding of the vector nature of kinematical concepts, Am. J. Phys. 73, 921 (2005).

[32] P. Barniol, G. Zavala, and C. Hinojosa, Students' difficulties in interpreting the torque vector in a physical situation, AIP Conf. Proc. 1513, 58 (2013).

[33] R. A. Serway and J. W. Jewett, Physics for Scientists and Engineers (Cengage Learning, Mexico, 2008).

[34] L. C. McDermott and P. Shaffer, Tutorials in Introductory Physics (Pearson Education, Buenos Aires, 2001).

[35] See Supplemental Material at http://link.aps.org/ supplemental/10.1103/PhysRevSTPER.10.010121 for the Test of understanding of vectors (TUV).

[36] R. W. Chabay and B. A. Sherwood, Matter and Interactions: Volume 2: Electric and Magnetic Interactions (Wiley, Hoboken, NJ, 2011).

[37] L. C. McDermott, Oersted Medal Lecture 2001: Physics education research-The key to student learning, Am. J. Phys. 69, 1127 (2001).

[38] E. Redish, Millikan Lecture 1998: Building a science of teaching physics, Am. J. Phys. 67, 562 (1999).

[39] R. R. Hake, Interactive-engagement versus traditional methods: A six-thousand-student survey of mechanics test data for introductory physics courses, Am. J. Phys. 66, 64 (1998). 